\title{
Effects of tramadol with tiletamine/zolazepam-xylazine as anaesthesia in cats
}

\author{
Lin $\mathrm{Li}^{1 \mathrm{a}}$, Jing Dong ${ }^{1 \mathrm{a}}$, Dezhang $\mathrm{Lu}^{2}$, Sheng Jiang ${ }^{3}$, Dongqi Lin ${ }^{3}$, Honggang Fan $^{3}$ \\ ${ }^{1}$ Shenyang Agricultural University, College of Animal Husbandry and Veterinary Medicine, Shenyang, China \\ ${ }^{2}$ Northwest A\&F University, College of Veterinary Medicine, Yangling, China \\ ${ }^{3}$ Northeast Agricultural University, College of Veterinary Medicine, Harbin, China \\ ${ }^{a}$ These authors have contributed equally to the development of this paper. \\ Corresponding author: Honggang Fan. Joint first author: Jing Dong \\ Received September 29, 2012 \\ Accepted March 19, 2013
}

\begin{abstract}
This study was conducted to investigate the effect of the combination of tiletamine/zolazepamxylazine-tramadol (TZXT) in cats and to compare its efficacy as an anaesthetic technique with tiletamine/zolazepam-xylazine (TZX). Fourteen healthy thirteen-month-old cats of both sexes were immobilized by TZXT or TZX on two different occasions. The cats' scores for immobilization, analgesia and baseline physiological indicators, heart rate, respiratory rate and rectal temperature, were recorded before and $5,10,15,20,30,40,50,60,80,100$ and 120 min after administration of TZXT or TZX. Animals in both groups became laterally recumbent within $3 \mathrm{~min}$ and physiological indicators changed after administration. These changes were within biologically acceptable limits; these measurements did not differ between the treatments. Induction and recovery quality in animals was better with TZXT than with TZX. Sedation, analgesia, muscle relaxation and posture scores was higher in TZXT group. During recovery, cats in TZXT group were more conscious than cats in TZX group. This study showed that in cats, addition of tramadol (T) to TZX improved the quality of anaesthetic induction and increased the analgesic effect without adversely affecting the physiological indicators. Thus tiletamine/ zolazepam-xylazine-tramadol shows to be more suitable for cat anaesthesia.
\end{abstract}

Felines, anaesthesia combination, physiological variables

Considering their unique temperament, relatively small size, dramatic stress response, and idiosyncratic drug metabolism, cats have always been an anaesthetic challenge. Sterilization clinics may operate in remote locations without access to inhalant anaesthetic (Cistola et al. 2004). The ideal injectable anaesthetic drugs should produce excellent hypnosis, muscle relaxation, analgesia and no adverse cardiorespiratory and physiological effects.

Tiletamine-zolazepam (TZ) has been used in veterinary medicine to facilitate handling of animals, especially TZ which has been used alone or in combination with other anaesthetic agents (Selmi et al. 2003; Aydilek 2007; Raušer et al. 2008). Xylazine(X), an $\alpha_{2}$-adrenoreceptor agonist, has been combined with tiletamine-zolazepam to increase its anaesthetic and analgesic effects and to reduce the dose of tiletamine-zolazepam required to induce satisfactory anaesthesia. However, their use is associated with adverse effects including hypertension and unwanted persistence of pharmacological effects (Lemke 2004). Tramadol is a centrally acting analgesic that is structurally related to codeine and morphine (Shilo et al. 2008). It acts as a weak m-opioid agonist coupled with inhibition of synaptic reuptake of serotonin and norepinephrine, achieving spinal modulation of pain and preventing impulses reaching the brain (Kongara et al. 2009). It had been used in several species, including cats (De et al. 2008; Paulo et al. 2008). But to the authors' knowledge, there are no data available about the use of tramadol in combination with TZX used in cats.

It was hypothesized that tramadol in combination with TZX might produce greater depth and duration of analgesia than TZX only. The aim of this study was to evaluate the

Address for correspondence:

Prof. Honggang Fan

College of Veterinary Medicine,

Northeast Agricultural University,

Harbin 150030, China
Phone: 86-0451-55190470

Fax: 86-0451-55190470

E-mail address: fanhonggang2002@163.com

http://actavet.vfu.cz/ 
anaesthetic and analgesic effects of tiletamine-zolazepam in combination with xylazine and tramadol (TZXT) in cats and to determine the magnitude of the changes in some basic physiological variables.

\section{Materials and Methods}

In total, fourteen cats of both sexes $13.3 \pm 2.5$ months old of a weight of $2.4 \pm 0.2 \mathrm{~kg}$ were used in the experiment that was approved by the Animal Care and Use Committee of the Shenyang Agricultural University (SYAU20120105). Before experiment, all cats were determined to be in good physical condition based on physical examination and complete blood count. Food was withdrawn for $12 \mathrm{~h}$ prior to the start of the experiment; water was available. Each cat was allowed to acclimate to room temperature at $25^{\circ} \mathrm{C}$ for at least 30 min before experiment. Subsequently, the cats was caught in a net and weighed. The rectal temperature (RT), heart rate (HR) and respiratory rate (RR) were measured by noninvasive monitor (Datex-OhmedaS/5TM, Datex-Ohmeda Division Instrumentarium Corp, Helsinki, Finland). The heart rate was determined by counting heart beats for 1 min using a stethoscope placed at the lower left lateral thoracic wall and RR was counted from thoracic excursions for $1 \mathrm{~min}$.

TZXT was administered by using a hand held syringe with an intended dose of $2.4 \mathrm{mg} \cdot \mathrm{kg}^{-1} \mathrm{TZ}$ (Zoletil ${ }^{\circledR} 100$, Virbac corporation, Carros, France), and $0.36 \mathrm{mg} \cdot \mathrm{kg}^{-1}$ xylazine (Rompun, Bayer, Leverkusen, Germany), with 0.8 $\mathrm{mg} \cdot \mathrm{kg}^{-1}$ tramadol (Tramal $^{\circledR} 100$; Grunenthal GmbH, Aachen, Germany) into muscles of the caudal thigh region. TZX was administered by using a hand held syringe with an intended dose of $2.4 \mathrm{mg} \cdot \mathrm{kg}^{-1} \mathrm{TZ}$ (Zoletil ${ }^{\circledR} 100$, Virbac corporation, Carros, France), and $0.36 \mathrm{mg} \cdot \mathrm{kg}^{-1}$ xylazine (Rompun, Bayer, Leverkusen, Germany) into muscles of the caudal thigh region. After baseline values (time 0) for physiological variables were collected, cats were immediately given the intramuscular injection drug combination; physiological variables were then recorded at 5 , $10,15,20,30,40,50,60,80,100$ and $120 \mathrm{~min}$. After immobilization, they were placed in the dorsal recumbency position on an operating table and an ophthalmic ointment was placed in the eyes to prevent corneal drying.

Scores for sedation, analgesia and muscle relaxation were assigned at each time point according to the criteria described in Table 1 and vomiting, excitement, and apnoea was monitored. Behavioural changes were evaluated by recording the cats' posture. A pin prick with a 22-gauge hypodermic needle was the method used to assess analgesia. The sequence of needle pricking was front limb along the radial-ulnar area followed by the perineal

Table 1. Criteria used to score anaesthetic effects of tiletamine/zolazepam-xylazine-tramadol or tiletamine/zolazepamxylazine in cats

\begin{tabular}{lll}
\hline Criteria & Score & Observation \\
\hline Sedation score & & Normal \\
1 & Mild sedation (recumbent, head down, strong palpebral reflex, normal eye position) \\
2 & Moderate sedation (recumbent, head down, moderate palpebral reflex, partial ventromedial eye rotation) \\
3 & Profound sedation (recumbent, head down, palpebral reflex absence, complete ventromedial eye rotation) \\
Analgesia score (analgesia evaluation via needle prick) & Normal (productive flight response) \\
1 & Mild (exaggerated movements of limbs and trying to get up) \\
2 & Moderate (slight movements of the limbs and trying to get up) \\
3 & Profound (lack of response) \\
0 & Normal resistance to open the mouth and leg tone \\
1 & The jaw can be opened, but there is still some resistance and mild leg tone \\
2 & Little resistance to open the mouth and obvious leg relaxation \\
3 & Profound relaxation of jaw and leg tone
\end{tabular}


area near the base of the tail. Any gross purposeful movement of the limb or body in reaction to the needle pricking was interpreted as lack of analgesia, and the scores of analgesia were evaluated and recorded. Analgesia evaluations occurred immediately after cardiorespiratory evaluation. All analgesia evaluations occurred in the same order at each time point.

Induction and anaesthesia times in each group were compared between groups using Student's $t$-test. Physiological variables were analyzed by means of ANOVA for repeated measures to evaluate changes within each group and between two different treatments. The scores for immobilization and analgesia (sedation, analgesia, muscle relaxation, posture) were compared using Mann-Whitney's test. All statistical analyses were performed using SPSS 13.0 (SPSS - Statistical Product and Service Solutions 13.0, SPSS Incorporation, Chicago, Illinois). A probability level of $5 \%(P<0.05)$ was considered significant. All values are reported as mean \pm standard deviation.

\section{Results}

After drug administration, animals showed signs of dissociative anaesthesia characterized by mydriasis and licking of the nose. Cats in both groups rapidly became laterally recumbent within $3 \mathrm{~min}$. Recovery was characterized by slow movements of the eyes, twitching of the ears and attempts to lift the head. Induction, anaesthesia and recovery times are recorded in Table 2 . Anaesthesia and recovery times were significantly longer using TZXT compared to TZX; induction time was not significantly different between two treatments. Vomiting, excitement, apnoea, or abnormal behaviour were not observed in any of the cats. In the TZXT treatment, animals were more conscious during recovery; ataxia and uncontrolled manners were rarely observed. There was no significant difference in physiological variables between two different treatments (Table 3).

Table 2. Induction, anaesthesia and recovery time in cats with tiletamine/zolazepam-xylazine-tramadol or tiletamine/ zolazepam-xylazine

\begin{tabular}{lccc}
\hline Doses & Induction time & Anaesthesia time & Recovery time \\
\hline TZXT & $2.19 \pm 0.88$ & $52.42 \pm 11.79^{\#}$ & $25.43 \pm 7.99^{\#}$ \\
TZX & $2.25 \pm 0.57$ & $41.64 \pm 11.46^{\#}$ & $19.61 \pm 8.98^{\#}$
\end{tabular}

Data expressed as mean $( \pm \mathrm{SD})$ values (minutes), \#- significant $(P<0.05)$ difference between groups. TZXT- tiletamine/ zolazepam-xylazine-tramadol, TZX- tiletamine/zolazepam-xylazine

Scores of sedation and analgesia in TZXT group were significantly compared to TZX group at $40 \mathrm{~min}$ after drug administration. Muscle relaxation and posture were significantly lower at 50 and $60 \mathrm{~min}$ in TZX group (Table 4).

\section{Discussion}

In our study, both TZXT and TZX produced complete immobilization for cats. Alexis et al. (2004) also reported that tiletamine/zolazepam $\left(2.8-5.6 \mathrm{mg} \cdot \mathrm{kg}^{-1}\right)$ and xylazine $\left(1.1-2.2 \mathrm{mg} \cdot \mathrm{kg}^{-1}\right)$ resulted an induction period of $4 \pm 1 \mathrm{~min}$ and recovery time of $108 \pm$ $24 \mathrm{~min}$ in cat anaesthesia. The doses of tiletamine/zolazepam and xylazine used in our study were much lower. Tiletamine/zolazepam-xylazine-tramadol had a quicker induction and better recovery compared with TZX. This result is most likely due to the prolonged sedative effect of tramadol. In this study the addition of tramadol increased significantly the duration of antinociception, which suggests that the combination of TZXT will be useful not only in induction of anaesthesia but also for surgery of short duration.

In cats anaesthetized with TZXT or TZX combination, HR was changed immediately after drug administration, and an anticholinergic agent was not used, but there was no 

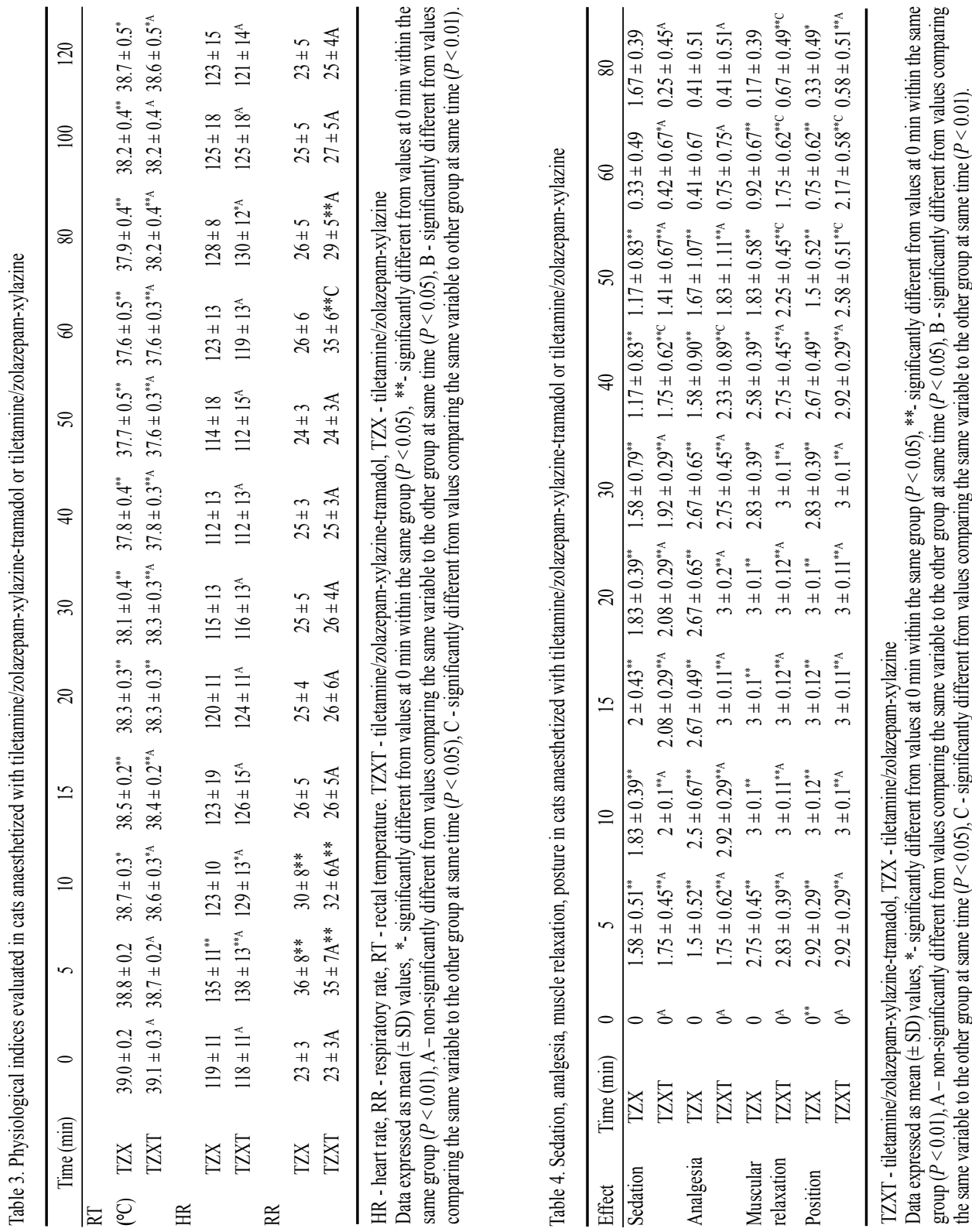

significant difference between two different treatments. The results of this study indicated that tramadol produced a less pronounced cardiovascular depression effects, the results also suggested that the positive chronotropic effects of TZ probably counterbalance temporarily and partially the bradycardic effect of xylazine (Witte et al. 2001; Selmi et al. 2003). Similar respiratory rates (RR) were observed in both groups, the results of this study also suggested that tramadol caused no significant additional respiratory depressant effect in 
cats. In this study, the RT of both groups decreased immediately after the injection of TZXT or TZX, possibly owing to loss of thermoregulatory control following administration of alpha-two adrenoreceptor agonists. Further, the decrease in RT may be due to generalized sedation, decrease in metabolic rate, muscle relaxation, and central nervous system depression produced by alpha-two adrenoreceptor agonists (Doherty et al. 1988).

When both treatments were compared, we observed that the degrees of sedation, analgesia, muscle relaxation, posture were higher in TZXT group. This could be attributed to the effect of tramadol $\left(0.8 \mathrm{mg} \cdot \mathrm{kg}^{-1}\right)$ in association with TZX which extends the anaesthetic time when compared to TZX alone. This also could explain TZXT had a longer recovery time than TZX because of the prolonged sedative effect of TZXT compared to TZX.

This study showed that TZX associated with tiletamine caused adequate immobilization characterized by rapid induction, adequate analgesia and muscle relaxation with no complications in cats.

\section{Acknowledgments}

This study was supported by the National Natural Science Foundation of China (Grant No. 31001092) and Special Research Fund for Doctoral Program of Universities of China (Grant No. 20102325120016). The editoral help from Dr. Hui Zhang in Northeast Agricultural University is appreciated.

\section{References}

Alexis M, Cistola, Francis J 2004: Anesthetic and physiologic effects of tiletamine, zolazepam, ketamine, and xylazine combination (TKX) in feral cats undergoing surgical sterilization. J Feline Med Surg 6: 297-303

Aydilek N 2007: Comparison between xylazine-tiletamine-zolazepam and fentanyl-tiletamine-zolazepam anaesthetic combinations on plasma oxidative status in sheep. Acta Vet Brno 76: 573-578

Cistola AM, Golder FJ, Centonze LA, McKay LW, Levy JK 2004: Anesthetic and physiologic effects of tiletamine, zolazepam, ketamine, and xylazine combination (TKX) in feral cats undergoing surgical sterilization. J Feline Med Surg 5: 297-303

De Sousa AB, Santos ACD, Schramm SG 2008: Pharmacokinetics of tramadol and O-desmethyltramadol in goats after intravenous and oral administration. J Vet Pharmacol Ther 31: 45-51

Doherty, TJ 1988: Physiologic effects of alpha 2-adrenergic receptors. J Am Vet Med Assoc 192: 1612-1614

Kongara K, Chambers P, Johnson CB 2009: Glomerular filtration rate after tramadol, parecoxib and pindolol following anaesthesia and analgesia in comparison with morphine in dogs. Vet Anaesth Analg 36: 86-94

Lemke KA 2004: Perioperative use of selective alpha-2 agonists and antagonists in small animals. Can Vet J 45: 475-480

Paulo VM, Steagall, Polly M 2008: Antinociceptive effects of tramadol and acepromazine in cats. J Feline Med Surg 10: 24-31

Raušer P, Lexmaulová L, Srnec R, Lorenzová J, Kecová H, Crha M, Fichtel T, Novotná R, Dvořák M, Nečas A 2008: Changes of vital parameters after administration of butorphanol during tiletamine-zolazepam-ketaminexylazine anaesthesia for joint surgery in miniature pigs. Acta Vet Brno 77: 251-256

Selmi AL, Mendes GM, FigueiredoI JP 2003: Chemical restraint of peccaries with tiletamine/zolazepam and xylazine or tiletamine/zolazepam and butorphanol. Vet Anaesth Analg 30: 24-29

Shilo Y, Britzi M, Eytan B 2008: Pharmacokinetics of tramadol in horses after intravenous, intramuscular and oral administration. J Vet Pharmacol Ther 31: 60-65

Witte JD, Schoenmaekers B, Sessler DL 2001: The Analgesic efficacy of tramadol is impaired by concurrent administration of ondansetron. Anesth Analg 92: 1319-1321 